\title{
Priming inverso: efectos contrarios en el comportamiento del consumidor
}

\author{
Laura Estefanía GARCíA DíAz ${ }^{1}$ \\ Universidad Pontificia Bolivariana
}

Recibido: 20/05/2014

Aceptado: 23/12/2014

\begin{abstract}
Resumen
Es coherente afirmar que con análisis y planeación, una campaña, pieza o táctica publicitaria deba resultar efectiva, pero ¿qué pasaría si estuvieran ocasionando comportamientos contrarios en el consumidor? La investigación que da lugar a este artículo se centró en comprender por medio de una revisión documental y entrevistas en profundidad con expertos, el efecto priming inverso, fenómeno que genera este tipo de reacciones. Si bien la bibliografía rastreada indica que no se ha documentado este fenómeno como tal en Latinoamérica, las implicaciones que podría tener su conocimiento para incrementar el número de campañas publicitarias efectivas son inminentes.
\end{abstract}

Palabras clave: Efecto priming, efecto priming inverso, memoria implícita, comportamiento, consumidor.

\section{Opposite effects at consumers behaviour}

\begin{abstract}
It is consistent to assert that with analysis and planning; a campaign, ad, or an advertisement tactic, should be effective, but $¿$ what if it would be causing an opposite behaviour on the consumer? Research leading to this article, focused on understanding through a documentary review and interviews with experts, the reverse priming effect, which makes such reactions happen. While the tracked literature indicates that phenomenon has not been documented in Latin America, implications of its knowledge to the improvement of effective advertisement campaigns are imminent.
\end{abstract}

Keywords: Priming effect, reverse priming effect, implicit memory, behaviour, consumer.

${ }^{1}$ Publicista de la Universidad Pontificia Bolivariana, Medellín - Colombia, integrante del Semillero de Investigación Demiurgo de la misma universidad, espacio académico en el cual se gestó la idea del proyecto que además de ser desarrollado como trabajo de grado, da origen al artículo. Email: lauraegarciadiaz@gmail.com. 


\section{Introducción}

Las tácticas de las estrategias publicitarias activan en el consumidor un conjunto de asociaciones mentales que tienen que ver directamente con sus aprendizajes previos y pueden lograr que se genere un comportamiento coherente con el que se está proponiendo; esto se puede atribuir a un efecto priming. Sin embargo, en algunas ocasiones, estas tácticas afectan automática e inconscientemente la decisión del consumidor, ocasionando que éste actúe en forma contraria, éste tipo de reacción se debe a un fenómeno de priming inverso.

Para comprender este último y su incidencia en la publicidad, es necesario comenzar por contextualizar de dónde viene y por qué ocurre.

\section{Sistemas de memoria}

La memoria hace referencia a toda codificación, almacenamiento y recuperación de la información aprendida y se clasifica en: memoria de corto y largo plazo.

La memoria de corto plazo se puede definir como la memoria reciente, tiene una duración aproximada de 60 segundos y tal y como lo afirma Ignacio Morgado Bernal (2005) "[...]es susceptible a las interferencias, puesto que logra que el recuerdo se desvanezca fácilmente"2, es el caso de recordar durante un momento el número telefónico al que se realizará una llamada y posteriormente olvidarlo.

La memoria de largo plazo, como su nombre lo indica, se encarga de recuperar aprendizajes de horas, días, meses e incluso años antes. Ésta a su vez se compone de una memoria explícita o declarativa y una memoria implícita o no declarativa; la primera se refiere a todos los recuerdos que pueden ser evocados de manera voluntaria o consciente, la segunda por el contrario, comprende la información que se recupera de manera no consciente.

La memoria implícita o no declarativa es la memoria del "cómo" y se relaciona con el proceso de aprendizaje de habilidades, por medio de ella se recuperan recuerdos que influencian el comportamiento actual pero de los cuales el individuo no es consciente; por ejemplo, si una persona se encuentra tocando un instrumento, implícita y automáticamente está trayendo la información del momento en el que aprendió, sin embargo este proceso no es percibido.

Es precisamente dentro de ésta donde se desarrollan los efectos de priming y priming inverso.

\section{Efecto priming}

El priming es un efecto de la memoria implícita que consiste en que la exposición a un estímulo, influye en la respuesta a uno posterior, generando un efecto de facilitación que según aclaran Tulving \& Schacter (1992) se puede generar incluso cuando el individuo no recuerda haber sido expuesto a dicho estímulo, así, una sola exposición durante unos segundos a una palabra, imagen, sonido, entre otros, puede ocasionar efectos posteriores de recordación que pueden ser identificados hasta un año después. Este término pertenece a la psicología cognitiva y no tiene una traducción precisa al español.

2 Morgado Bernal, I. (2005): "Psicobiología del aprendizaje y la memoria", Cic: cuadernos de información y comunicación, 10, 222. 
El priming se comenzó a estudiar en profundidad a finales de los años 80 cuando se identificó como una opción alternativa de aprendizaje para los pacientes con amnesia, puesto que trabajaba desde la memoria implícita y no desde la explícita donde se encontraba la lesión, este hallazgo desencadenó un gran número de experimentos para caracterizar el fenómeno.

El progreso en entender la relación entre el priming y el cerebro, ha sido facilitado por la interacción entre estudios cognitivos de individuos con las funciones de memoria intactas, las investigaciones neuropsicológicas de pacientes con desórdenes de memoria y la investigación con la neuroimagen funcional. ${ }^{3}$

Según el procesamiento del primer estímulo (definido como primador o facilitador del segundo), el priming puede ser perceptual o semántico. En el priming perceptual, se refleja la actividad del sistema de representación perceptual del individuo que según Eugenia Razumiejczyk, Alfredo O. López Alonso y Guillermo Macbeth (2008): “[...] procesa y representa la información sobre la forma y la estructura, pero no sobre el significado u otras propiedades asociativas de los estímulos"4, es decir, que en el caso tal de que el estímulo primador fuese un anuncio, el priming perceptual se daría sobre sus características físicas y no sobre su contenido, pues en éste último se centra el priming semántico.

Stewart Shapiro (1999) se dedicó a analizar la influencia de la publicidad en los mecanismos que están más allá de la voluntad del ser humano. Cuatro estudios demostraron que la respuesta sesgada por la exposición incidental a un anuncio se debe a influencias inconscientes, "Mientras unas persona centra su atención en una cosa, otra información que no es el objeto de atención puede ser procesada, afectando la actitud hacia el anuncio y hacia la marca." 5

\section{Efecto priming inverso}

Los efectos priming son invertidos cuando el no consciente del consumidor percibe una fuente potencial de persuasión, en este caso su comportamiento resulta opuesto al sugerido en la táctica publicitaria. Al igual que el priming, este efecto ocurre dentro de la memoria implícita, lo que quiere decir que estos cambios en el comportamiento no son advertidos por la persona.

En el comienzo de las investigaciones que dieron a conocer este fenómeno, como cita Frank Kardes (1988): "Walster y Festinger (1962) demostraron que un agente de

\footnotetext{
3 Schacter, D. L., \& Buckner, R. L. (1998): "Priming and the Brain", Neuron, 20, 192.

4 RazumiejczyK, E., López Alonso, A. O., \& Macbeth, G. (2008): "El Efecto de Priming y sus Variantes Experimentales", Publicación virtual de la facultad de Psicología y Psicopedagogía de la USAL, (18). http://www.usal.edu.ar/archivos/psico/otros/el_efecto_de_priming_y_sus_ variantes_experimentales.pdf. Web visitada el 15/02/2012.

5 Shapiro, S. (1999): "When an Ad's Influence Is beyond Our Conscious Control : Perceptual and Conceptual Fluency Effects Caused by Incidental Ad Exposure", Journal of Consumer Research, 26 (1), 16. http://www.jstor.org/stable/10.1086/209548?origin=JSTOR-pdf. Web visitada el 16/02/2012.
} 
influencia es más persuasivo si el intento por persuadir no es obvio." ${ }^{\prime 6}$. Un mensaje es más persuasivo cuando es escuchado incidentalmente que cuando es intencionalmente dirigido al perceptor.

Posteriormente, en 1994, Timothy Wilson y Nancy Brekke hablan sobre la contaminación mental, que es el proceso por el cual una persona tiene una respuesta no deseada debido al procesamiento mental que es inconsciente e incontrolable. Se habla de lo "no deseado" refiriéndose a que en el momento en que una persona está tomando una decisión sobre algo, preferiría no ser influenciado, "La mayoría preferiría que sus decisiones de consumo no fueran afectadas por la publicidad de productos, porque

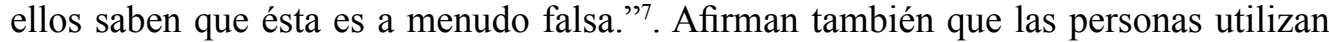
estrategias para proteger su mente de las influencias y se ejemplifica con el fenómeno del zapping.

Los sesgos o influencias a una persona se pueden dar por dicha contaminación mental: La persona sabe y tiene claro que no quiere ser influenciada, pero de igual manera no se da cuenta de que esto sucede y no lo puede evitar. Este fenómeno se puede ilustrar de la siguiente manera: Un entrenador de fútbol está escogiendo el nuevo capitán del equipo y tiene claro que su posición debe ser neutra, pero uno de los jugadores es su ahijado; finalmente su decisión, más allá de su voluntad, se verá influenciada.

Según Wilson D. \& Brekke (1994) Las personas no están prevenidas de las fuentes de contaminación por las siguientes razones:

- Ellos no creen que puedan ser influenciados por cosas como ruidos distractores o preguntas capciosas.

- Creen que los contaminantes son potencialmente influenciadores, pero a su vez piensan que tienen estrategias efectivas para contrarrestarlo. Como poner una aplicación en su explorador para evitar la exposición a publicidad intrusiva indeseada.

- Se cree también que el contaminante existe y lo encuentran frecuentemente, que no pueden contrarrestarlo, pero piensan que las consecuencias que trae éste no tienen importancia.

Algunos estudios (Wilson D \& Brekke, 1994) mostraban que hacer conscientes a las personas de que las están influenciando, hace que éstas ajusten sus respuestas; sin embargo incrementando el nivel de conciencia, no desaparece el efecto priming, sino que éste se revierte, generando un efecto de contraste.

En el año 1999, Jack Glaser y Mahzarin Banaji se centran un poco más en el fenómeno. "Se teoriza que el efecto priming inverso refleja una corrección automática

${ }^{6}$ KARDES, F (1988): "Spontaneous Inference Processes in Advertising: The Effects of conclusion Omission and Involvement on Persuasion", Journal of Consumer Research, 15, 225. http://www.jstor.org/stable/10.2307/2489527. Web visitada el 16/01/2013.

7 Wilson, T., \& BREKKe, N. (1994): "Mental contamination and mental correction- Unwanted influences on judgment and evaluation", Psychologycal Bulletin, 116 (1), 117. http://people. virginia.edu/ tdw/wilson\%26brekke.1994.pdf. Web visitada el 08/01/2013. 
para la influencia de desviación del prime" ${ }^{\text {. }}$. El estímulo con el que se realiza el priming influencia la decisión del individuo de una manera coherente, pero bajo ciertas condiciones, éste puede llevar a decisiones que contrastan y son lo opuesto del prime. Strack y Hannover (como se cita en Glaser \& Banaji, 1999) argumentan: "[...] La conciencia de la influencia de los primes, lleva a medidas correctivas que pueden generar efectos de contraste." da espontáneamente cuando el priming es atrevido, pero no cuando es sutil; es decir, cuando el estímulo de priming o su potencial para afectar la decisión es más evidente, existe mayor posibilidad de que surja un contraste; también se puede dar cuando las capacidades cognitivas y motivacionales de la persona hacen que ésta recuerde el prime. Dada la naturaleza interventiva del fenómeno, los priming extremos negativos, tienen efectos positivos y viceversa.

Afirman además (J Glaser \& Banaji, 1999) que es posible que otras investigaciones tengan resultados similares y que se encuentren escondidos o perdidos en los archivos de la investigación en psicología. Finalmente, se dice que las personas son inconscientemente vigilantes de la información que los puede influenciar y sin darse cuenta pueden generar estrategias correctivas.

Para el 2003, Jack Glaser continuó profundizando en el tema. Con sus investigaciones, encontró que cualquier actitud puede ser obtenida indirectamente sin intención alguna y que la percepción, categorización, evaluación y corrección, pueden ocurrir sin procesos conscientes. También planteó bajo qué condiciones se da la evaluación automática. Se originaron reportes de que los priming no siempre funcionaban, algunos sólo lo obtenían cuando el estímulo prime era oscurecido al final (fundido a negro) pero no cuando era presentado de manera normal; otros no lo obtenían cuando el tiempo de exposición era de $1000 \mathrm{~ms}$ o cuando los estímulos primes representaban objetos hacia los cuales los individuos tenían actitudes débiles (Jack Glaser, 2003). Pero en lo que coincide en este reporte Glaser, con Glaser \& Banaji (1999) es que el priming inverso, definido como priming en la dirección opuesta de lo que estaba planteado en el prime, persiste cuando éstos son extremos, atrevidos.

Los resultados de investigaciones recientes (Laran et al., 2011), afirman que existe un fenómeno llamado: Corrección automática en contra de la persuasión, este proceso consiste en que cuando los consumidores encuentran una fuente no deseada, los procesos mentales y comportamientos están incitados a corregir su influencia potencial. Las respuestas a este estímulo incluyen contraargumentos, evaluaciones negativas sobre la fuente de persuasión, y preferencias inversas en los comportamientos.

\footnotetext{
${ }^{8}$ Glaser, J, \& BanaJi, M. R. (1999): "When fair is foul and foul is fair: reverse priming in automatic evaluation", Journal of personality and social psychology, 77 (4), 670. http://www. ncbi.nlm.nih.gov/pubmed/10531668. Web visitada el 02/03/2013.

9 Glaser, J, \& BanaJ, M. R. (1999): "When fair is foul and foul is fair: reverse priming in automatic evaluation", Journal of personality and social psychology, 77 (4), 672. http://www. ncbi.nlm.nih.gov/pubmed/10531668. Web visitada el 02/03/2013.
} 


\section{Aplicaciones del efecto priming inverso en la publicidad}

A partir de lo anterior y como hallazgos de la investigación que da lugar a este artículo, es posible hablar sobre los campos de acción en relación con la publicidad, con el fin de hacer tangibles las posibles aplicaciones del efecto priming inverso como herramienta para el desarrollo de estrategias publicitarias efectivas.

\subsection{Marca y eslógan}

La marca es mucho más que un diferenciador de un producto o servicio, se desarrolla a partir de las estrategias de branding, y debe construir un capital de marca que según David Aacker (1996) se define como: "Conjunto de cualidades (y responsabilidades) vinculadas con el nombre y los símbolos de una marca que se agrega (o resta) al valor proporcionado por un producto o servicio a una empresa y (o) al cliente de esa empresa." 10 . Además de un sistema de identidad de marca, personalidad, entre otros conceptos propios del branding como herramienta para generar marcas rentables y con valor.

Sin embargo, aquella definición corresponde a la percepción de los profesionales del mundo del marketing y la publicidad; para las personas, en general, la marca es simplemente una etiqueta que tiene el fin de diferenciar un producto de sus similares. Con esta aclaración, se hace posible comprender por qué las marcas producen un efecto priming en los consumidores y no un efecto priming inverso, esto tiene que ver directamente con que al percibirla como una característica cualquiera, no es catalogada por el no consciente del individuo como un estímulo de fuente persuasiva, por lo tanto, no se da la corrección. No quiere decir esto que las estrategias de branding estén exentas, pero al menos actualmente, en la medida en que el consumidor continúe con esta opinión sobre las marcas, que está ligada a su cultura, a sus aprendizajes, a sus puntos de vista, no se verán efectos contrarios en la aplicación de tácticas publicitarias de branding.

Con el eslógan sucede lo contrario, si bien los consumidores no toman las marcas como vehículos de persuasión, las frases publicitarias no pasan tan desapercibidas, pues es más lógico para ellos asimilar que el único objetivo de éstas es vender; Eulalio Ferrer afirma: "La eficacia de un mensaje publicitario se mide no solo por el interés que despierta, sino por los grados de sugestión persuasiva y utilidad factible que contenga o transmita"11. Únicamente cuando las frases poseen un alto índice de creatividad, es posible llegar a evadir la noción de su objetivo intrínseco. Por las razones expresadas y según los experimentos de Laran et al. (2011) los slogan desatan en el consumidor actitudes contrarias en su comportamiento, consecuencia de un efecto priming inverso.

\footnotetext{
${ }^{10}$ Aacker, D. A. (1996): El Éxito de su Producto está en la Marca, México, Prentice Hall Hispanoamérica, S.A.

11 Ferrer, E. (1994): El Lenguaje de la Publicidad, México, Fondo de Cultura Económica de España, 1995, 235.
} 


\title{
5.2 Marketing olfativo
}

Con la tendencia de neuromarketing que comenzó a desarrollarse hace algunos años con el fin de encontrar bases neurológicas que pudiesen aumentar la efectividad de los esfuerzos de las compañías en inversiones publicitarias, se identificaron los olores como herramientas que desencadenan varias reacciones en el individuo pues según el Especialista en Marketing José Ruiz Pardo (2012): "De los cinco sentidos que tenemos los seres humanos sin lugar a dudas el olfato es el que más rápidamente es capaz de evocar sensaciones y sentimientos."12. A partir de este hallazgo, se ha implementado el tema del marketing de olfato para la creación de «odotipos» de las empresas. La publicidad entonces, está logrando experiencias de marca que cada vez más involucran los sentidos en el proceso de generación de recuerdos y asociación de emociones, así lo ratifica la empresa española Marketing Olfativo en su sitio web: "[...] consiste en utilizar aromas específicos en un entorno de negocio con el fin de suscitar las emociones, y con ello influir sobre los comportamientos del consumidor [...]."13

Con respecto al fenómeno de priming y priming inverso, es claro que se puede presentar en cualquier modalidad de sentidos, la particularidad con el olfativo radica en que según Koenig, Bourron, \& Royet (2000) los olores no pueden ser fácilmente clasificados en características perceptuales, pero a su vez, la fuerte asociación que tienen estos a recuerdos y evocación de emociones, hacen que resulte siendo un estímulo primador muy efectivo; la pregunta es ¿qué pasa entonces cuando el no consciente del consumidor descubre que al entrar a un punto de venta están tratando de persuadirlo con un odotipo? ¿Cómo se mide la efectividad de esta táctica? ¿Se testeó de manera científica previamente a su implementación? Es factible que en muchos casos se esté presentando un efecto priming inverso que impida un real incremento en las ventas del almacén en cuanto a la utilización de esta táctica se refiere.

\subsection{Sitios web, diagramación y "packaging”}

Según afirma la empresa SEO Colombia:

\begin{abstract}
En Colombia, una página web es una herramienta muy valiosa para abrirse camino hacia nuevos negocios, para posicionarse en el mercado local y para generar una recordación de marca entre potenciales clientes. No por nada, los presupuestos de las grandes empresas para publicidad en Internet ha aumentado año tras año. [...] Toda empresa debe entender que tener una página web no solamente es un canal publicitario, sino también un medio para mejorar el servicio al cliente, investigar mercados o crear estrategias logísticas. ${ }^{14}$
\end{abstract}

Se resalta la importancia de los sitios web como instrumento estratégico para el posicionamiento de empresas y la diagramación como complemento indispensable para la efectividad de la misma, pues logran generar cambios en las decisiones de los consumidores, más aún, cuando se examina la acción del efecto priming y efecto

${ }^{12}$ Ruiz PARdo, J., “Aquí Huele a Marca”, Neuromarketing Spain. http://neuromarketingspain. blogspot.com/2012/02/aqui-huele-marca.html. Web visitada el 25/08/2013.

13 Marketing Olfativo. http://www.aromarketing.es/. Web visitada el 25/08/2013.

14 SEO Colombia. http://www.seocolombia.com/diseno-de-paginas-web-en-colombia/. Web visitada el 25/08/2013. 
priming inverso: Cualquier cambio sutil en el diseño de la página web que incorpore un elemento cuya función sea primar, puede producir diferentes elecciones, dirigir focos de atención e influenciar preferencias tanto en consumidores novatos en el segmento, como en expertos (Mandel \& Johnson, 2002). Pero tanto así como puede incitar estos comportamientos, dependiendo de las características del estímulo primador, también puede cancelarlos.

Exactamente lo mismo sucede con el tema de packaging, pues trabaja de la mano de la diagramación y gracias al éxito de esta y a la pertinencia de la información, es factible considerarlo "el vendedor silencioso".

\subsection{Contexto cognitivo y afectivo de los anuncios}

Todo anuncio se encuentra inmerso en un contexto, todo contexto tiene una significancia que genera un impacto ya sea emocional o cognitivo, y toda significancia influye directamente en la interpretación que realiza el consumidor sobre la pieza, ya sea consciente o no conscientemente. En todo medio siempre existirá la posibilidad de que diferentes contenidos generen valencias positivas o negativas en el individuo target, ya sea en una revista o prensa, si el anuncio es precedido por una noticia; en televisión un programa o incluso otro spot, en radio, medios digitales, publicidad exterior, entre otros.

El contexto de la publicidad puede condicionar o activar determinados atributos en los lectores, y guiar sus interpretaciones del producto en el anuncio. Estas interpretaciones pueden terminar en cambiar las creencias del consumidor acerca de la marca. ${ }^{15}$

Se dice entonces que el priming cognitivo, condiciona los atributos de un producto y determina el tipo de interpretación dada la información de éste en el anuncio; y el priming afectivo, provoca reacciones emocionales en la audiencia, influyendo en las evaluaciones que ésta hace del anuncio y posteriormente, de la marca. Éstos descubrimientos, son de gran influencia para el área de la publicidad, ya que se afirma en ellos que el mismo anuncio puede producir diferentes efectos, dependiendo del contexto donde se encuentre inmerso.

Para la segmentación de públicos se hace indispensable saber que los experimentos realizados por Yi, Ph.d. en Marketing de la Universidad de Stanford (1990), arrojan resultados que aseguran que el estado afectivo en el que se encuentre el consumidor en el momento de ser expuesto al anuncio, es determinante en la actitud hacia éste y se concluye que mientras el priming cognitivo afecta la actitud hacia la marca y las intenciones de compra, el priming afectivo interviene en la actitud hacia el anuncio y de igual manera en las intenciones de compra.

15 Yi, Y. (1990): “Cognitive and affective priming effects of the context for print advertisements", Journal of Advertising, 19 (2), 40. http://www.jstor.org/stable/10.2307/4188762. Web visitada el 16/02/2012. 
Si el contexto es intervenido con pre-exposiciones que actúen como facilitadores para que el mensaje funcione al momento del consumidor encontrar el anuncio, será un satisfactorio acierto. A su vez, siempre que se da un efecto priming existe la posibilidad de que éste termine en un priming inverso y los esfuerzos generen resultados contradictorios.

\subsection{Propaganda política}

En la política, cada persona, cada candidato, representa un conjunto de ideas que al «venderse» a la sociedad generan una cantidad de adeptos que se identifican con estas gracias a un universo de variables que tienen que ver con sus objetivos, su cultura, sus aprendizajes, sus creencias, etc. Muchas veces, las ideas son bien recibidas pero el individuo no transmite confianza a la comunidad, lo que finalmente evita que éste sea elegido. Trabajar estrategias de priming puede aumentar la confianza en la política, siempre y cuando se encuentre en coherencia con lo que dicta la ética. Además, por medio de este, sería posible cambiar los imaginarios internos de las personas con respecto a las evaluaciones políticas, cambios en los juicios de importancia, e incluso, fortalecer la ideología de una candidato teniendo en cuenta el contexto en el que se genera. Según Michele Claibourn (2008), "El priming puede predecir los resultados de un líder si por medio del priming se refuerza la asociación entre un concepto determinado y su evaluación en la memoria de largo plazo." ${ }^{\prime 6}$. En todo proceso se debe realizar el debido testeo, pues en caso de que un estímulo de pre-exposición sea percibido como persuasivo en la memoria implícita, los resultados podrían ser devastadores.

\subsection{Publicidad social}

Para enmarcar a grandes rasgos el término y en qué difiere de la publicidad comercial, es necesario distinguir que en tanto la última actúa como un impulsador para el consumo, la publicidad social dirige todos sus objetivos a generar un cambio. Con respecto a esta vertiente de la publicidad, Luis Bassat plantea:

Conseguir que las amas de casa consoliden su buena imagen hacia un producto cualquiera del supermercado es siempre motivo de orgullo y satisfacción para un publicitario. Pero conseguir que bajen los índices de accidentes mortales de jóvenes en la carretera un fin de semana, lo es aún más. La publicidad para instituciones sigue los mismos principios y las mismas reglas que la publicidad para productos de consumo, pero su grado de trascendencia es mucho mayor.

Las problemáticas sociales son temas que afectan la convivencia y/o el bienestar de la comunidad, al ser Colombia un país del tercer mundo, estas situaciones se ven potenciadas por la pobreza, el cuestionamiento sobre la transparencia de la utilización de los recursos públicos, entre muchos otros. Parte de estos recursos se destina a fortalecer y educar a la sociedad para disminuir este tipo de problemas, pero su efectividad no es evidente, las personas siguen actuando contrario a lo que se les dice, aún cuando

${ }^{16}$ Claibourn, M. P. (2008): "Making a Connection: Repetition and Priming in Presidential Campaigns", The Journal of Politics, 70 (04), 1143. 
el tema afecta directamente su salud, su familia, incluso su vida; son contados los esfuerzos publicitarios que han logrado resultados significativos.

Ahora bien, son muchos los estudios y análisis que se han realizado sobre las causas de los comportamientos en la sociedad, con cada campaña se apela a un recurso diferente con el fin de incrementar las acciones positivas como resultado, pero ¿qué posibilidad existe de que estas respuestas contrarias se estén generando por un efecto priming inverso? Se reitera que este fenómeno ocurre sin ninguna intervención consciente, ¿Y si confirmándolo se procediera a determinar estrategias de priming previamente testeadas? Problemáticas como el control de adicciones (tabaquismo, alcohol, droga, juego, etc), embarazos adolescentes, enfermedades de transmisión sexual, accidentes de tránsito, accidentes de trabajo, violencia intrafamiliar; en caso de que esta herramienta se implementara, posiblemente se hablaría de cifras diferentes, de cambios reales en los comportamientos y de probablemente un incremento sin precedentes en la calidad de vida de los ciudadanos.

\section{Conclusión}

Si bien la documentación del fenómeno de priming inverso no es tan reciente como las últimas investigaciones, aún falta mucho por conocer sobre él y sus implicaciones, pues según el rastreo realizado, aún no hay compañías que hayan documentado métodologías de trabajo basadas en la corrección de efectos priming inversos, y dado el abanico de usos que tiene el efecto priming como tal, es perfectamente concebible que se puedan presentar en cada uno de ellos. Este hecho resalta la importancia tanto de impulsar los testeos antes, durante y después de campaña, así como de invertir para potenciar la investigación científica en publicidad, con el fin de entender la lógica del consumidor y su comportamiento como un ser biológico, desarrollando fundamentos teóricos complementarios en los que sea posible construir herramientas en un futuro; de esta manera, cada día más se encaminará la profesión a transformarse en una ciencia.

Si este efecto se puede gestar ante cualquier estímulo que el no consciente de un consumidor precise como persuasivo, quiere decir que existe la posibilidad de que surja ante cualquier pieza (visual, auditiva, experiencial, etc), método o táctica de cualquier especialidad que tenga por objetivo vender, ya sea productos, servicios o ideas.

Teniendo en cuenta la utilidad ya analizada del efecto priming en las tácticas publicitarias, el hecho de conocer y comprender que existe un efecto contrario, puede garantizar de una manera más tangible que la comunicación realmente sea efectiva y que no genere actitudes no deseadas, por el contrario, con la buena utilización de estímulos primadores como herramienta metodológica para el desarrollo de estrategias y piezas, comenzará una nueva etapa en donde los índices de efectividad serán evidentes, medibles y satisfactorios.

De la mano de la conclusión anterior, es posible inferir que se generará una optimización en las inversiones y presupuestos que manejan las empresas anualmente para marketing y publicidad, pues las campañas serán realizadas con bases 
neurocientíficas testeadas trabajando en conjunto con los métodos actuales de análisis de segmentos, investigación de mercados, entre otros.

Conocer el efecto priming inverso además, le brinda a los publicistas la capacidad/ opción de cambiar comportamientos en el consumidor, estos cambios pueden ser en cuanto a acciones de compra que favorezcan a las empresas y a la economía del país, como en cuanto a los objetivos de la publicidad social donde su efectividad implicará que las decisiones personales tomadas incrementen los índices de calidad de vida, sana convivencia y bienestar, todo esto a partir de una inhibición del efecto inverso que podría estar generando actualmente la interpretación del mensaje a nivel de memoria implícita.

Una idea no es buena o mala, lo es el uso que se le de y las intenciones o intereses con los que se utilice. Desde la ética entonces, podría decirse que la inhibición del efecto priming inverso para asegurar un efecto priming satisfactorio en la publicidad es un tema a tratar con responsabilidad y respeto, analizando en profundidad los casos en su contexto, la pertinencia de la herramienta en los mismos y las repercusiones que pueda traer tanto para el individuo como para la sociedad. Ante todo trabajar siempre con información verídica, honesta y comprobable que tenga en cuenta la cultura en la que se encuentra inmersa y que no desequilibre el bienestar del consumidor, por el contrario, que ayude siempre a potenciarlo y mejorar su calidad de vida. En el panorama legislativo en Colombia, la viabilidad de la toma de acciones con respecto al efecto priming inverso en la publicidad, se relaciona directamente con el respeto a los principios axiológicos discutidos anteriormente, analizados desde el ámbito de los derechos de las personas, los consumidores, las empresas, los niños y la salud. Claro está que en el momento en el que las campañas comiencen a ser testeadas para evitar que se de el fenómeno, deberán establecerse unas regulaciones específicas e incluso un instituto de control especializado a partir de estudios científicos, para que las implicaciones de su resultado (efecto priming) estén en coherencia con los lineamientos ya mencionados. Debe proteger entonces la libertad, respetar las poblaciones susceptibles como lo es la infancia y actuar conforme a las restricciones que poseen los temas de alcohol y tabaco.

Por último, a estas conclusiones se suma el impacto que podría tener en la investigación publicitaria orientada a sentar bases científicas, pues esta investigación ha abierto ventanas a nuevos objetos de análisis, básicamente cada aplicación que tiene el conocimiento del priming inverso en la comunicación persuasiva, se convierte en una línea a desarrollar.

\section{Bibliografía}

AACKer, D. A. (1996): El Éxito de su Producto está en la Marca, México, Prentice Hall Hispanoamérica, S.A.

Bassat, L. (1993): El Libro Rojo de la Publicidad, Madrid, Editorial Espasa Calpe S.A. Claibourn, M. P. (2008): "Making a Connection: Repetition and Priming in Presidential Campaigns", The Journal of Politics, 70 (04), 1142-1159. 
Ferrer, E. (1994): El Lenguaje de la Publicidad, México, Fondo de Cultura Económica de España, 1995.

Glaser, J, \& BANAJI, M. R. (1999): "When fair is foul and foul is fair: reverse priming in automatic evaluation", Journal of personality and social psychology, 77 (4), 66987. http://www.ncbi.nlm.nih.gov/pubmed/10531668. Web visitada el 02/03/2013

Glaser, J. (2003): "Reverse priming: Implications for the (un) conditionality of automatic evaluation", The psychology of evaluation: Affective processes in Cognition and Emotion, 87-108. http://scholar.google.com/scholar?hl=en\&btnG= Search\&q=intitle:Reverse+Priming: + Implications + for + the + (un) + conditionality $+o$ f+automatic+evaluation\#0. Web visitada el 16/01/2013.

KARDES, F. (1988): "Spontaneous Inference Processes in Advertising: The Effects of conclusion Omission and Involvement on Persuasion", Journal of Consumer Research, 15, 225-233. http://www.jstor.org/stable/10.2307/2489527. Web visitada el 16/01/2013.

Koenig, O., Bourron, G., \& Royet, J. P. (2000): "Evidence for separate perceptive and semantic memories for odours: a priming experiment", Chemical senses, 25 (6), 703-8. http://www.ncbi.nlm.nih.gov/pubmed/11114148. Web visitada el $08 / 01 / 2013$.

Laran, J., Dalton, A. N., \& Andrade, E. B. (2011): “The Curious Case of Behavioral Backlash: Why Brands Produce Priming Effects and Slogans Produce Reverse Priming Effects", The Journal of Consumer Research, 37 (6), 999-1014. http:// faculty.haas.berkeley.edu/eandrade/p12_LaranDaltonAndrade_JCR2010.pdf. Web visitada el 15/02/2012.

Mandel, N., \& Johnson, E. (2002): "When web pages influence choice: Effects of visual primes on experts and novices", Journal of Consumer Research, 29 (2), 235 245. http://www.jstor.org/stable/10.1086/341573. Web visitada el 16/01/2013.

Marketing Olfativo. http://www.aromarketing.es/. Web visitada el 25/08/2013.

Morgado Bernal, I. (2005): "Psicobiología del aprendizaje y la memoria", Cic: cuadernos de información y comunicación, 10, $221-233$.

Razumiejczyk, E., López Alonso, A. O., \& Macbeth, G. (2008): "El Efecto de Priming y sus Variantes Experimentales", Publicación virtual de la facultad de Psicología y Psicopedagogía de la USAL, (18). http://www.usal.edu.ar/archivos/psico/otros/ el_efecto_de_priming_y_sus_variantes_experimentales.pdf. Web visitada el $1 \overline{5} / 02 / 20 \overline{12}$.

Ruiz Pardo, J., "Aquí Huele a Marca”, Neuromarketing Spain. http:// neuromarketingspain.blogspot.com/2012/02/aqui-huele-marca.html. Web visitada el 25/08/2013.

Schacter, D. L., \& Buckner, R. L. (1998): "Priming and the Brain", Neuron, 20, 185195.

SEO Colombia. http://www.seocolombia.com/diseno-de-paginas-web-en-colombia/. Web visitada el 25/08/2013.

Shapiro, S. (1999): "When an Ad's Influence Is beyond Our Conscious Control: Perceptual and Conceptual Fluency Effects Caused by Incidental Ad Exposure", Journal of Consumer Research, 26 (1), 16-36. http://www.jstor.org/stable/10.1086/ 209548? origin=JSTOR-pdf. Web visitada el 16/02/2012. 
Tulving, E., \& Schacter, D. L. (1992): "Priming and memory systems", Neuroscience Year - Supplement 2 to the Encyclopedia of Neuroscience, 130-133. http://www. ncbi.nlm.nih.gov/pubmed/15093145. Web visitada el 28/08/2013.

Wilson, T., \& BrekKe, N. (1994): "Mental contamination and mental correctionUnwanted influences on judgment and evaluation", Psychologycal Bulletin, 116 (1), 117-142. http://people.virginia.edu/ tdw/wilson\%26brekke.1994.pdf. Web visitada el $08 / 01 / 2013$.

YI, Y. (1990): "Cognitive and affective priming effects of the context for print advertisements", Journal of Advertising, 19 (2), 40-48. http://www.jstor.org/ stable/10.2307/4188762. Web visitada el 16/02/2012. 